\title{
Dust along the SNe lines-of-sight
}

\author{
Nancy Elias-Rosa* \\ Max-Planck-Institut für Astrophysik, Karl-Schwarzschild-Str. 1, D-85748 Garching bei \\ München, Germany \\ E-mail: nelias@mpa-garching.mpg.de
}

\section{J. E. Beckman}

Instituto de Astrofísica de Canarias, C/Vía Láctea s/n, E-38200, La Laguna, Tenerife, Spain.

E-mail: jeb@iac.es

\section{Stefano Benetti}

INAF - Osservatorio Astronomico di Padova, Vicolo dell'Osservatorio 5, I-35122 Padova, Italy.

E-mail: stefano.benetti@oapd.inaf.it

\section{Enrico Cappellaro}

INAF - Osservatorio Astronomico di Padova, Vicolo dell'Osservatorio 5, I-35122 Padova, Italy. E-mail: enrico.cappellarodoapd.inaf.it

\section{Massimo Turatto}

INAF - Osservatorio Astrofisico di Catania, Via S.Sofia 78, I-95123 Catania, Italy.

E-mail: massimo.turatto@oact.inaf.it

The determination of the extinction in different directions in the Galaxy and in external galaxies is of paramount importance in several fields of astrophysics. The determination of the extinction law provides clues to the nature of the Inter/Circum-Stellar Medium (ISM/CSM), specifically to that of the dust particles. Recent studies have shown that the extinction curves toward a few, highly-reddened SN Ia have values of $\mathrm{R}_{V}$, the ratio of total-to-selective extinction, significantly lower than the canonical 3.1. Whether this is the case only for high extinction objects is not clear yet. Clarifying this is extremely important both for understanding the nature of the supernovae $(\mathrm{SNe})$ and for the calibration of SNe Ia as cosmological standard candles. Thus, in the context of our present knowledge of dust, we discuss here the information that we can obtain from these cases of highly extinguished SNe Ia, i.e. the possibility of deriving the average size of the dust grains along their lines-of-sight, which, in all cases, is smaller than the average dust grain size found in the Galaxy, and offer a complementary study of the behaviour of the NaID absorption lines in the SNe spectra. If the interstellar NaID doublet is present in the spectra we find that the supernova light is at least reddened by $E(B-V)=0.11 \times E W(N a I D)$.

Supernovae: lights in the darkness (XXIII Trobades Científiques de la Mediterrània) October 3-5 2007

Mao, Menorca, Spain

\footnotetext{
${ }^{*}$ Speaker.
} 
Dust is a ubiquitous feature of the cosmos, impinging directly or indirectly on most fields of modern astronomy. Its study is a highly active area of current research. The determination of the extinction by dust is one of the most crucial ingredients in the calibration of SNe for their use as distance indicators. Indeed the correction for this effect has been studied in the past but no universally accepted solutions have been found. Often the analysis of the reddening in nearby SNe has given hints of anomalous reddening. A practical solution is to remove obviously reddened objects from the statistical samples, which reduces the problem but does not solve it. This text aims to give an overview of this phenomenon and of how it can affect our interpretation of the $\mathrm{SNe}$ observations.

\section{Dust}

Astrophysical dust is believed to be a mixture of particles with different sizes, shapes and compositions. They account for only $1 \%$ of the total interstellar (hereafter IS) mass (the rest is gas). In a typical spiral galaxy, dust is concentrated in the galactic disc, producing dark lanes in edge-on spirals. In contrast, there is relatively little dust in elliptical galaxies. Within the disc, much of the dust is associated with the spiral arms. Its distribution is highly inhomogeneous, and in the Galaxy it is usual to find high density clumps of dust and gas with typical sizes in the range $1-50 \mathrm{pc}$ which are traditionally termed clouds. In general terms dust is found in the interstellar medium (hereafter ISM), which fills the space between the stars, and also in circumstellar medium (hereafter CSM) which is closely associated with an individual star.

Today dust particles play a fundamental role in the explanation of many phenomena: they are implicated in star formation, in the formation of molecules in IS space, drive the mass loss in stars at the end of their lives and are vital in the formation of planets.

Despite their small size, ranging from about $3.5 \times 10^{-4} \mu \mathrm{m}$ to about $10 \mu \mathrm{m}$, dust grains are able to reduce the radiation emitted by stars mainly in the visual and ultraviolet bands. The dust grains can both absorb and scatter the incoming radiation. While the scattered part is only deviated towards a different line of sight, the absorbed radiation heats the grains making them re-emit the energy as diffuse thermal energy. This process is more efficient towards UV wavelengths. As a result, we experience a deficit of UV radiation and an excess of IR radiation along a line of sight dominated by IS dust. For this reason, the extinction process is normally referred to as reddening.

The size distribution of IS grains is a consequence of the balance of constructive and destructive processes.

There are two mechanisms for the growth of dust particles: (i) coagulation, as a consequence of grain-grain collisions, and (ii) mantle growth when gas-phase atoms accumulate on a grain surface. The former process redistributes the grain mass, the latter create new grain material and increases the dust-to-gas ratio. In a dense cloud, the differential reddening $\mathrm{R}_{V}$ is often enhanced as a consequence of coagulation. This mechanism reduces the number of small grains, increases the number of large grains and it has a systematic effect on the shape of the extinction curve ([1] and references therein).

There are two main mechanisms that destroy grains: (i) sputtering and (ii) grain-grain collisions ([2] and references therein). The former can be chemical or physical. Chemical sputtering arises when gas atoms reach the grain surface at low velocity and react with the atoms on the grain 

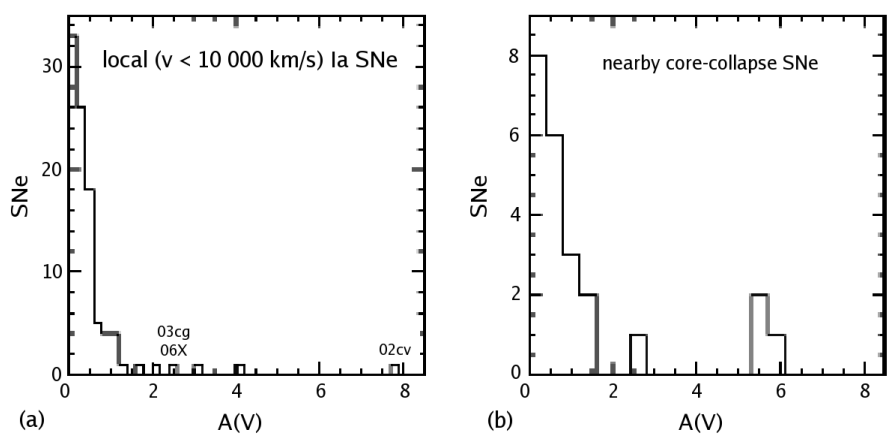

Figure 1: Histogram of the host galaxy extinction of nearby Type Ia SNe (a) and core collapse $\mathrm{SNe}$ (b). The $\mathrm{SNe}$ discussed in this work are marked in the figure (from [4]).

forming molecules that are desorbed. If the particle impinging on the grain has high kinetic energy the sputtering is physical, the removal of surface atoms by energetic impact.

Both sputtering and grain-grain collisions destroy the grains in the diffuse ISM when they are affected by a supernova shock wave ([3] and references therein). Destruction by shocks is more efficient in the diffuse ISM than in dense clouds because the shock is decelerated when it encounters a cloud with density higher than that of the diffuse ISM.

\section{Dust and supernovae}

It is known that the SNe play an important role in generation and evolution of the ISM, and that, in the context of the present study, they contribute the $8 \%$ of dust content of the ISM. We can use the high luminosities of the SNe to help with the analysis of the reddening law and the study of the diversity in the physical characteristics of the dust causing extinction. Typically, dust extinction is larger in the case of core collapse $\mathrm{SNe}$, as we can see in Figure 1, because they are preferentially located in dust-rich environments, and smaller for Type Ia SNe, particulary for those exploding in elliptical galaxies, which are, in general, largely dust-free. Nonetheless, there are several examples of Type Ia SNe showing moderate to high extinction as we will see in the examples below.

It is clear that $\mathrm{SNe}$ are a very "hot" topic in astrophysics. Nevertheless a large number of open questions remain with regard to the nature of these exploding stars, the explosion mechanisms, and the degree of extinction suffered by the SN light on its path to us. This extinction reduces the precision of our measurements of the energy of the explosion, required to understand the physics fully, and limits our ability to use $\mathrm{SNe}$ as distance indicators. However it has been shown (e.g. [5]) that thermonuclear $\mathrm{SNe}$ (SNe Ia) are a reasonably homogeneous (though not perfect) class of emitters; just for this reason they are widely used as distance indicators. This property, their homogeneity, can be exploited to derive the properties of the dust along the line of sight toward the $\mathrm{SNe}$ and the associated extinction laws. By measuring the colour excess of a large sample of Type Ia SNe, it is possible to see that the majority of SNe Ia suffer an extinction of less than 0.40 mag (see Figure 1). In this case the changes of the SED (spectral energy distribution) are small. The few cases of objects with very high extinction have a fundamental role and can help to shed light on the behaviour of the extinction law obeyed by the ISM inside the host galaxies. 


\subsection{Extinction}

Observations give us a lot of information about IS grains. The study of depletion in the ISM, plus direct spectroscopy study allow us to investigate the composition of dust, and the data on extinction, scattering, polarization and emission give information about the distribution of grain sizes. Here we will concentrate mainly on the extinction phenomena (more detailed information about the other processes can be found in, e.g. [6]).

The extinction is most reliably determined using the "pair" method: comparing spectrophotometry of two stars of the same spectral type and luminosity class but unequal reddening.

The apparent magnitude of each star as a function of wavelength may be written:

$$
\begin{gathered}
m_{1}(\lambda)=M_{1}(\lambda)+5 \log d_{1}+A_{1}(\lambda) \\
m_{2}(\lambda)=M_{2}(\lambda)+5 \log d_{2}+A_{2}(\lambda),
\end{gathered}
$$

where $M, d$ and $A$ are the absolute magnitude, distance and total extinction respectively; indexes 1 and 2 correspond to the "reddened" and "comparison" stars. Assuming $M_{1}(\lambda)=M_{2}(\lambda)$ and $A(\lambda)$ $=A_{1}(\lambda) \gg A_{2}(\lambda)$, the magnitude difference $\Delta m(\lambda)=m_{1}(\lambda)-m_{2}(\lambda)$ is reduced to

$$
\Delta m(\lambda)=5 \log \left(\frac{d_{1}}{d_{2}}\right)+A_{\lambda}
$$

Normalization with respect to two standard wavelengths $\lambda_{1}$ and $\lambda_{2}$ allows us to eliminate the distance:

$$
E_{\text {norm }}=\frac{\Delta m(\lambda)-\Delta m\left(\lambda_{2}\right)}{\Delta m\left(\lambda_{1}\right)-\Delta m\left(\lambda_{2}\right)}=\frac{A_{\lambda}-A_{\lambda_{2}}}{A_{\lambda_{1}}-A_{\lambda_{2}}}=\frac{E\left(\lambda-\lambda_{2}\right)}{E\left(\lambda_{1}-\lambda_{2}\right)}
$$

where $E\left(\lambda_{1}-\lambda_{2}\right)$, the difference in extinction between the specified wavelengths, is termed the colour excess, for instance $\mathrm{E}(\mathrm{B}-\mathrm{V})=(\mathrm{B}-\mathrm{V})-(\mathrm{B}-\mathrm{V})_{0}$.

Because the reddened/unreddened star pair are very rarely at the same distance and, in fact, the stellar distances are usually poorly determined, the total extinction $\mathrm{A}_{\lambda}$ is not often computed directly. Instead, the stellar SEDs are usually normalized by the flux in a common wavelength region before computing the extinction. The usual choice for this is the optical V band. This normalization in the $\mathrm{V}$ band is usually named extinction curve, and it is related to the total extinction $\mathrm{A}_{\lambda}$ through the relation

$$
\frac{E(\lambda-V)}{E(B-V)}=\frac{A_{\lambda}-A_{V}}{E(B-V)}=R_{V}\left\{\frac{A(\lambda)}{A_{V}}-1\right\}
$$

where $R_{V}\left[\equiv \frac{A_{V}}{E(B-V)}\right]$ is the ratio of total-to-selective extinction.

The first to demonstrate a link between the UV and the optical/IR extinction in the sense that lines of sight with large $\mathrm{R}_{V}$ values tend to have low UV extinction, and viceversa, were [7] (hereafter CCM). CCM derived a mean extinction law of the form $A_{\lambda} / A_{V}=a_{x}+\left(b_{x} / R_{V}\right)$, in the range $0.12 \mu \mathrm{m}<\lambda<3.5 \mu \mathrm{m}$, which depends only on the parameter $\mathrm{R}_{V}\left(a_{x}\right.$ and $b_{x}$ are functions of $\lambda$ and $x=1 / \lambda)$. In other words the extinction curves of their sample constitute a one-parameter family, the parameter being $\mathrm{R}_{V}$. 


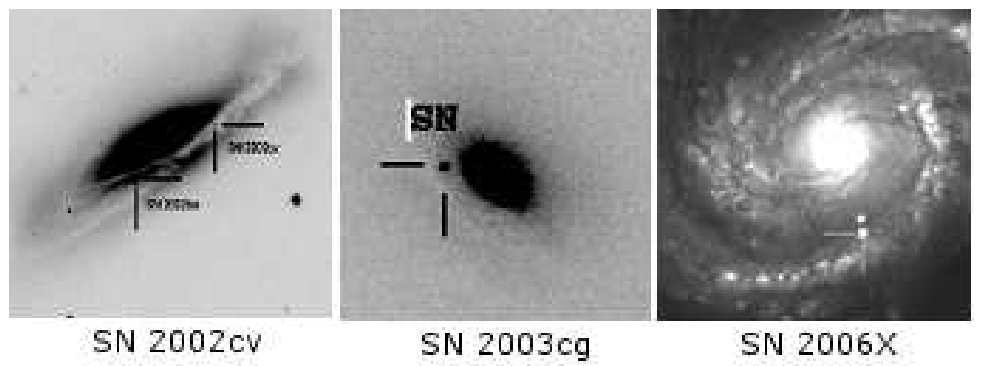

Figure 2: SN 2002cv ([13]), SN 2003cg ([14]) and SN 2006X ([15]) are three examples of high extinguished SNe with $R_{V} \ll 3.1$.

As noted above, in the Milky Way the observed dust properties vary from one direction to another. Our knowledge of the extinction law for dust in other galaxies is derived basically from the Large and Small Magellanic Clouds (LMC and SMC), where it has been possible to study the extinction for individual stars. Several lines of sight in the LMC appear to obey extinction laws similar to that for the Milky Way diffuse clouds, as measurements yield $\mathrm{R}_{V}=3.1$ in most cases $([8,9])$. In the SMC, stars in the "bar" region have extinction curves which appear to lack the $2175 \AA$ feature. In M31, [10] found an extinction law similar to the average Milky Way extinction curve but with the $2175 \AA$ feature somewhat weaker than in our Galaxy. The variation in the extinction properties seen in the Magellanic Clouds and M31 may be due to several factors, for example different environments (e.g. Star formation regions where large amounts of UV radiation and shocks are present) ([11]). For other galaxies where individual stars cannot be resolved, there are different approaches to determining the extinction curve for dust. The dust extinction law can be determined when a foreground galaxy overlaps a background galaxy, or using observations of the overall emission spectrum in starburst galaxies or from analysis of QSO spectra ([12]).

\subsection{Low total-to-selective extinction ratios for $\mathrm{SNe}$ Ia}

We pointed out above that normally dust extinction is smaller for Type Ia SNe because they explode in elliptical galaxies, which are, in general, almost dust-free. In these cases the changes of the SED are small and comparable to the uncertainties in the photometric calibration. Nonetheless, there are a few cases with very high extinction which are important because they can help to shed light on different extinction laws obeyed by the ISM within the host galaxies. Examples of this are SN 2002cv ([13]), SN 2003cg ([14]) and SN 2006X ([15]) (see Figure 2). For all of these we have used several methods to estimate the extinction between the observer and the SNe. The methods rely on the differential effects of extinction on the SED, and use either the SN colours and photometry, or spectra with good S/N. In both cases, we obtained more robust results by combining optical and IR observations (see each reference paper for a deeper discussion about these points). In any case, for all of these $\mathrm{SNe}$ we have found that $\mathrm{R}_{V}$ is considerably less than 3.1 (see Table 1).

As explained above, the total-to-selective extinction ratio, $\mathrm{R}_{V}$, has been well studied in the Milky Way yielding a mean value of $\mathrm{R}_{V}=3.1$. However, for a few directions, values ranging from $\mathrm{R}_{V} \sim 2$ to $\sim 5.5$ have been found (e.g. [16]). In other galaxies, we have relatively poor information about the extinction parameters. The studies of $\mathrm{R}_{V}$ from $\mathrm{SNe}$ have provided values ranging from 0.70 ([17]) to 3.1 ([18]). Since $\mathrm{R}_{V}$ is probably related to the average characteristics of dust grains 
Table 1: $R_{V}$ and $\mathrm{E}(\mathrm{B}-\mathrm{V})$ for the three cases of high extinction

\begin{tabular}{lccl}
\hline SN & $\mathrm{E}(\mathrm{B}-\mathrm{V})$ & $R_{V}$ & ref \\
\hline SN 2002cv & $5.12 \pm 1.09$ & $1.73 \pm 0.54$ & {$[13]$} \\
SN 2003cg & $1.33 \pm 0.11$ & $1.80 \pm 0.19$ & {$[14]$} \\
SN 2006X & $1.27 \pm 0.37$ & $1.56 \pm 0.30$ & {$[15]$} \\
\hline
\end{tabular}

that contribute to extinction along the line of sight, we may infer that small values of $\mathrm{R}_{V}$ correspond to a small mean size for the dust grains. This kind of grains must belong to diffuse clouds because studies in dense clouds confirm that these grains grow by coagulation, which implies a large $\mathrm{R}_{V}$ ([6]). From the works of [19] and [20], small values of $\mathrm{R}_{V}$ are measured in galaxies with well-defined dust lanes, and in fact the $\mathrm{SNe}$ we have studied are projected onto dust lanes of their host galaxies (see Figure 2).

Focusing on $\mathrm{SNe}$ Ia [5] summarized possible reasons why the total-to-selective extinction ratios in SN Ia are so small (without reaching any firm conclusions): (1) because of the different dust properties in the host galaxy with respect to the Galactic dust, (2) because the observed spread of (B-V) at maximum might be enlarged by observational errors, (3) the assumption of a unique intrinsic $(B-V)_{0}^{\max }$ for all Type Ia SNe could be invalid, (4) due to circumstellar dust with very particular optical properties. Some years later, [21] demonstrated from the study of 20 distant $\mathrm{SNe}$ Ia that on average, the optical properties of dust in distant galaxies are not drastically different from those of the Milky Way and that the variation of the $\mathrm{R}_{V}$ is not caused by circumstellar dust but by IS dust, because, if not, we would expect the measured $\mathrm{A}_{V}$ to be independent of galaxy morphology, which it is not the case, as shown in their Figure 6.

An alternative explanation was introduced by [22] who considers that dust in the immediate neighborhood of SNe Ia can explain the anomalous reddening seen in SNe Ia. This idea implies light echo scenarios where the light is scattered out of and into our line of sight. In that study [22] found that the light echo from a dust cloud with an inner radius $10^{16} \mathrm{~cm}$ would produce a significant reduction in $\mathrm{R}_{V}$. By comparing the panels of Figure 2 of [22], it is clear that $R_{\lambda}$ is reduced around the optical maximum if dust scattering effects are included.

However, there are difficulties with this interpretation. Firstly, the possibility of grain survival at such a small distances from a SN is low, and we would expect to observe other effects at later times such as a strong near-IR excess, time variation in the reddening, an anomalously small $\Delta m_{15}$, a significantly brighter late phase tail or broader spectral lines ([23]), which are not seen.

We conclude, therefore, that the low values of $\mathrm{R}_{V}$ we observe in the $\mathrm{SNe}$ are caused mainly by dust grains of small size in the ISM along the lines of sight. In general, most of the host galaxies have properties different from the Milky Way, and there are cases of SNe within the same host galaxy whose $\mathrm{R}_{V}$ is different (e.g. in NGC 3190 where we find $\mathrm{R}_{V} \overline{3} .1$ for SN 2002 bo and 1.7 for SN 2002cv, [13]). A hypothesis could include the possibility that the UV radiation from the SN explosions might have partially eroded the dust, increasing the number of small grains. An optical evidence of this was found in SN 2006X ([24]). This would imply that "circum-SN" dust is the cause of these low values of $\mathrm{R}_{V}$. However, this is not the only possibility; there are still some 
open questions in this explanation: Is this effect always "local", i.e. somehow related to the nature of the progenitor system, or due to the overall dust properties of the host galaxy? Has this not been detected previously just because high spectral resolution has not been used? Is the peculiar extinction law derived in the direction to these SNe Ia common to all the highly-reddened objects? Do low-reddening SNe Ia follow similar extinction laws? What is the effect on the calibration of nearby SNe Ia? Are the extinction laws the same at low and high redshift?

\subsection{Estimating the relative grain sizes along line of sights}

Having $\mathrm{R}_{\lambda}\left[\equiv \frac{A_{\lambda}}{E(B-V)}\right]$ from the extinction curves obtained along the line of sight to our three examples of highly reddened $\mathrm{SNe}$, we can estimate the characteristic grain size, relative to that in the Milky Way, which produced the observed extinction following the procedure outlined by [19] and [20].

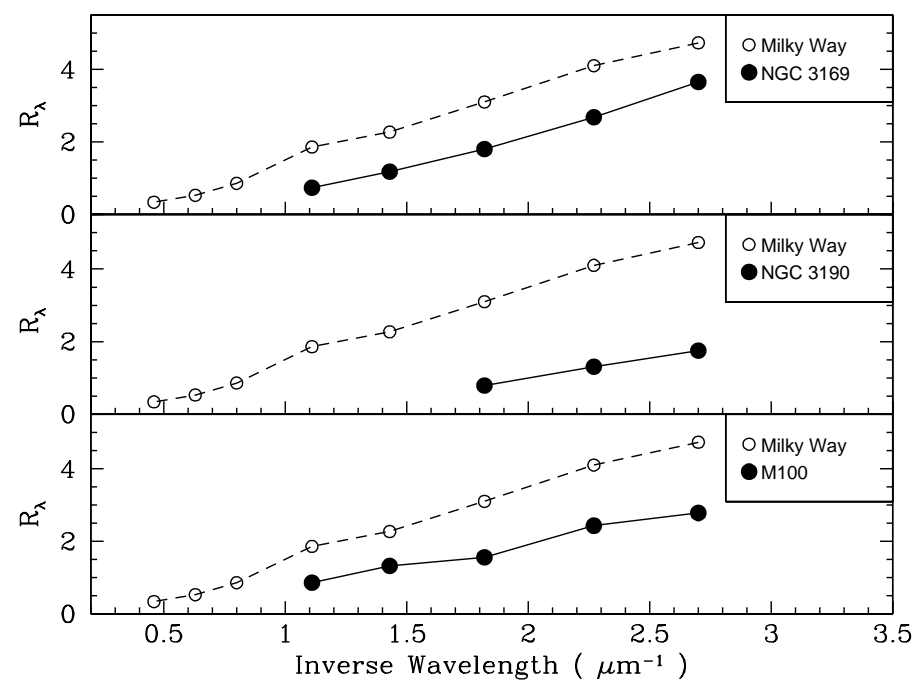

Figure 3: Extinction curves for the host galaxies in the direction to our three examples of highly extinguished SNe Ia (filled circles, solid lines) compared with the canonical curve for the Milky Way (open circles, dashed lines).

In Figure 3 we have plotted the $\mathrm{R}_{\lambda}$ values obtained from the extinction curves in the direction to our SNe with those for the Milky Way. This figure demonstrates that, on average, the extinction curves for these galaxies are virtually parallel to that of our Galaxy, and this should imply that the dust extinction properties in the extragalactic environment are similar to those of the Milky Way, although $\mathrm{R}_{V}$ is found to be smaller than the canonical value 3.1 of the Milky Way.

As stated above, assuming that the chemical composition of dust grains in the extragalactic environment is similar (on average) to that of our Galaxy, smaller $\mathrm{R}_{V}$ values imply that the dust grains causing IS reddening are smaller.

This allows us to compute the relative grain size in the sample galaxies by making use of available models of chemical composition and shape of the dust grains, such as that proposed by 
Table 2: Relative grain sizes.

\begin{tabular}{llc}
\hline SN & Host galaxy & $\langle a\rangle / a_{G}$ \\
\hline SN 2002cv & NGC 3190 & $0.52 \pm 0.06$ \\
SN 2003cg & NGC 3169 & $0.75 \pm 0.07$ \\
SN 2006X & M 100 & $0.71 \pm 0.07$ \\
\hline
\end{tabular}

[25]: a two-component model which consists of spherical graphite and silicate grains. This model assumes uncoated refractory particles having a power law size distribution $n(a)=n_{0} a^{-3.5}$, where "a" is the grain radius. As the present study is restricted to the optical regime, this model serves as a good approximation for this purpose.

To estimate the relative grain sizes we can use the extinction efficiency $\mathrm{Q}_{e x t}$, which is a function of a dimensionless size parameter defined by $X=2 \pi a / \lambda$ where $a$ is the grain radius. For small grains, $\mathrm{Q}_{e x t} \propto \lambda^{-1}$ i.e. for $\mathrm{X} \leq 1$. Since the extinction curve in the optical part of the spectrum varies proportionally to $\lambda^{-1}, \mathrm{Q}_{e x t}$ is proportional to $\mathrm{X}$. For a given $\mathrm{Q}_{e x t}$, the mismatch between the extinction curve for the Milky Way and those for our host galaxies is attributed to the difference in grain size between the host galaxies and the Milky Way.

Considering the definition of $X$ and the condition for small grains, we can write

$$
a \leq \frac{1}{2 \pi \lambda-1} \Rightarrow \frac{\langle a\rangle}{a_{G}} \leq \frac{\lambda_{G}^{-1}}{\lambda_{G}^{-1}+\Delta\left(\lambda^{-1}\right)}
$$

calling $\frac{\langle a\rangle}{a_{G}}$ the grain size (mean) of our host galaxies relative to the Milky Way grain size, $\lambda_{G}^{-1}$ is the inverse wavelength for the Milky Way, and $\Delta\left(\lambda^{-1}\right)$ is the difference between the inverse wavelengths of the two extinction curves (host galaxy and Milky Way), i.e. the quantity needed to bring the two extinction curves into coincidence.

Therefore, one can estimate the relative grain size by shifting the observed extinction curve along the $\lambda^{-1}$ axis until it best matches the Galactic extinction curve, in the sense that the extinction curve lying below the Galactic curve will correspond to smaller grain size relative to that of Galactic dust grains. The relative grain sizes derived for the lines of sight within the host galaxies are shown in Table 2. It must be stressed that the values reported here are not average values for those galaxies, as are those reported by [19] and [20], but specific to the line of sight to the SNe. Indeed, for NGC 3190 we have studied another line of sight in the direction to SN 2002bo which has provided a standard extinction curve (that is that both extinction curves, this one and that for the Milky Way, coincide), hence standard grain size. This points up how variable can be the extinction curve along different lines of sight within the same galaxy, and also confirms the finding by [19] and [20], that the properties of grains in the dust lane appear to be different from those of grains in the rest of the galaxy.

\subsection{Equivalent width of $\mathrm{Na} \mathrm{ID}$ and reddening in $\mathrm{SNe}$}

In the ISM, the dust is a relatively minor constituent of the gas clouds which mostly consists of gas. The gas properties have been determined by observing the absorption effects at ultraviolet, optical and infrared wavelengths, and the emissions at radio and millimetric wavelengths. By measuring IS absorption lines we can in principle obtain the total column density of the absorbing 
gas along the line of sight. This can, in turn, be related to the amount of IS dust although with caution because the gas to dust ratio in the ISM does vary. In first approximation however, the measurements of the EW of the narrow IS lines, in particular the Na ID doublet (5890.0, 5895.9 $\AA$ ), could give us a direct estimate of the reddening which is a crucial ingredient in the calibration of SNe. A first study using SNe was performed by [26] with low resolution spectra who found a linear relation $\mathrm{E}(\mathrm{B}-\mathrm{V})=0.25 \times \mathrm{EW}(\AA)$ over the whole range $0.0 \leq \mathrm{E}(\mathrm{B}-\mathrm{V}) \leq 1.0$. A similar study on high resolution spectra of $32 \mathrm{O}$ and early $\mathrm{B}$ stars was made by [27]. They confirmed the existence of a linear relation for Na ID and extended the work to higher reddening using the weaker K I doublet. Finally, [28] reviewed the [26] work increasing the sample with data for SNe Ia. They found that SNe Ia occupy a large area in the EW(Na ID) vs. E(B-V) plane and seem to favour two regions described by the linear relations: $E(B-V)=0.16 \times E W(N a I D)$ and $E(B-V)=0.51 \times E W(N a I D)$. This study was limited due to the use of low resolution spectra which, not only limit the accuracy of the measurements at low EW, but yield errors due to the blending of the two differently saturated doublet lines.

We reexamine the $\mathrm{EW}(\mathrm{Na} \mathrm{ID})$ vs. $\mathrm{E}(\mathrm{B}-\mathrm{V})$ relation using a larger sample of $\mathrm{SNe}$ of all types ([29]). The EW of NaI D and of the reddening both in the Milky Way and in SN host galaxies for a sample of $90 \mathrm{SNe}$ of all types were collected. The values of the EW(Na ID) come from direct measurements on the low resolution spectra of the Asiago Supernova Archive ([30]) or from literature. The $\mathrm{E}(\mathrm{B}-\mathrm{V})$ were determined in more ways. For SNe Ia we preferred to use determinations based on the colour curves at epochs between 30 and 90 days past maximum ([31]). For core collapse $\mathrm{SNe}$ the estimates are in general less reliable and less homogeneous. They come from comparison of colour curves of objects of similar classes, from the comparison of the SED with theoretical models and from studies of the high resolution component of the IS lines. In Figure 4 we show the complete distribution of $\mathrm{SNe}$ data in the $\mathrm{EW}(\mathrm{Na} \mathrm{ID}) \mathrm{vs}$. $\mathrm{E}(\mathrm{B}-\mathrm{V})$ plane which presents a wide scatter.

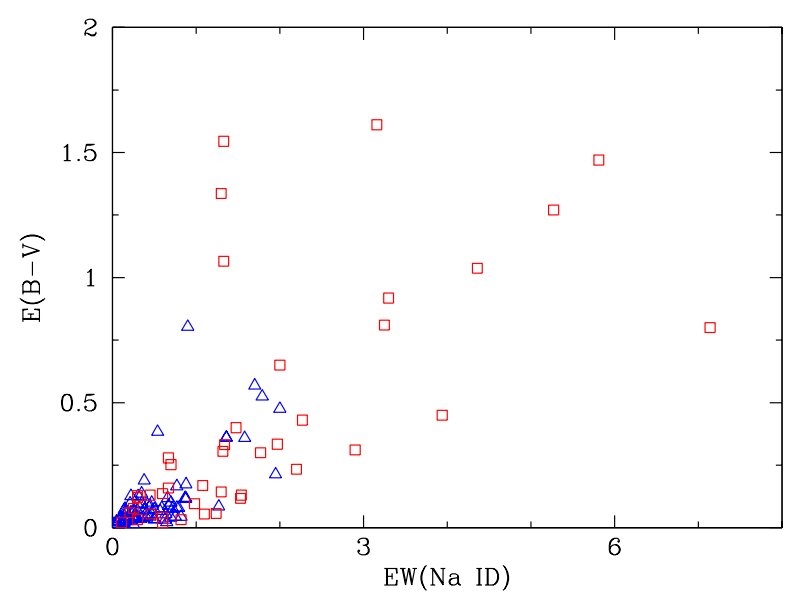

Figure 4: Relation between equivalent width of Na ID and reddening for the $90 \mathrm{SNe}$ of our sample. Triangles and squares correspond to measurements for the Milky Way and the SN host galaxies, respectively.

We discuss several possible causes of the observed distribution. 
1. Position, distance, inclination and type of the host galaxies: Since we are working with $\mathrm{SNe}$ in different host galaxies, we first consider the position and type of the host galaxies of those SNe with large values of EW(Na ID) or E(B-V) (see Figure 4) in order to explore effects which may affect the distribution. This test proved negative. There was no evidence for any dependence of the equivalent width or extinction on distance, galaxy type, galaxy inclination.

2. Variable dust-to-gas ratio: It is known that $\mathrm{EW}(\mathrm{Na} \mathrm{ID})$ is related to the column density of gas and $\mathrm{E}(\mathrm{B}-\mathrm{V})$ is related through $\mathrm{A}_{V}$ to the column density of dust grains. They are connected by the dust-to-gas ratio $\left(\rho_{d} / \rho_{g}\right)$, which depends on the chemical composition of the ISM, on the physical conditions of the clouds and on the depletion of their most abundant elements. This implies different values of $\rho_{d} / \rho_{g}$ even within the same galaxy and our knowledge of the average dust-to-gas ratio in other galaxies is still scanty. Studies of column densities of atomic hydrogen, molecular gas and grain populations are needed. Each $\mathrm{SN}$ of our sample represents not only a different host galaxy but also a different line of sight. The large dispersion of the points in the plane $\mathrm{EW}(\mathrm{Na}$ ID) vs. $\mathrm{E}(\mathrm{B}-\mathrm{V})$ proves the variation of $\rho_{d} / \rho_{g}$, but we might expect also to find more dispersion for large values, and this is not found.

3. Variable value of $R_{V}$ : To relate $\mathrm{E}(\mathrm{B}-\mathrm{V})$ with the optical depth of the dust, the parameter $\mathrm{R}_{V}$ has to be taken into account. As shown above, there are $\mathrm{SNe}$ for which $\mathrm{R}_{V}$ is lower than the standard, $\mathrm{R}_{V}=3.1$. So the wide range of possible values of $\mathbf{R}_{V}$ could be another source of dispersion but not the only one because for this effect, considering that $\mathrm{R}_{V}$ can range from 0.70 to 5.5, we would experience an even greater dispersion of the points in the plane $\mathrm{EW}(\mathrm{Na} \mathrm{ID})$ vs. $\mathrm{E}(\mathrm{B}-\mathrm{V})$, and this is not found either.

4. Saturated lines in multiple clouds: Line saturation can increase the scatter in Figure 4. The ideal way to derive column densities from the Na ID absorption lines is to record spectra at a sufficiently high resolution and good signal-to-noise ratio and to apply the curve of growth method (Figure 5, left). In Figure 4 we have superimposed a number of curves of growth due to identical gas clouds to cover the area of interest of the EW(Na ID) vs. E(B-V) plane (Figure 5, right). These curves were created by widening the scope of the relation found by [27] between $\mathrm{E}(\mathrm{B}-\mathrm{V})$ and $\mathrm{EW}(\mathrm{Na}$ ID) using the contributions of both components of the Na ID blended doublet.

It is clear that every point of the plane can be explained by a combination of various curves of growth due to clouds of different column density. These curves are clearly an approximation because we have assumed constant values of a dust-to-gas ratio and $\mathrm{R}_{V}$ ([27]).

To verify this, we measured separately the EW of both lines of the doublet from the high resolution spectra taken for some of the most extinguished $\mathrm{SNe}$ in the figure and available from our archive or from literature. It is known that if the doublet ratio is close to 1.1, this implies that the lines are saturated, while a ratio of around 2.0 implies that the lines are unsaturated indicating the presence of warmer clouds (e.g. [33]). The measured doublet ratio of SNe in the lower highlighted region of the plane EW(Na ID) vs. E(B-V) of Figure 6 is close to the unsaturated value and the other points have ratios close to 1.1. Our interpretation 

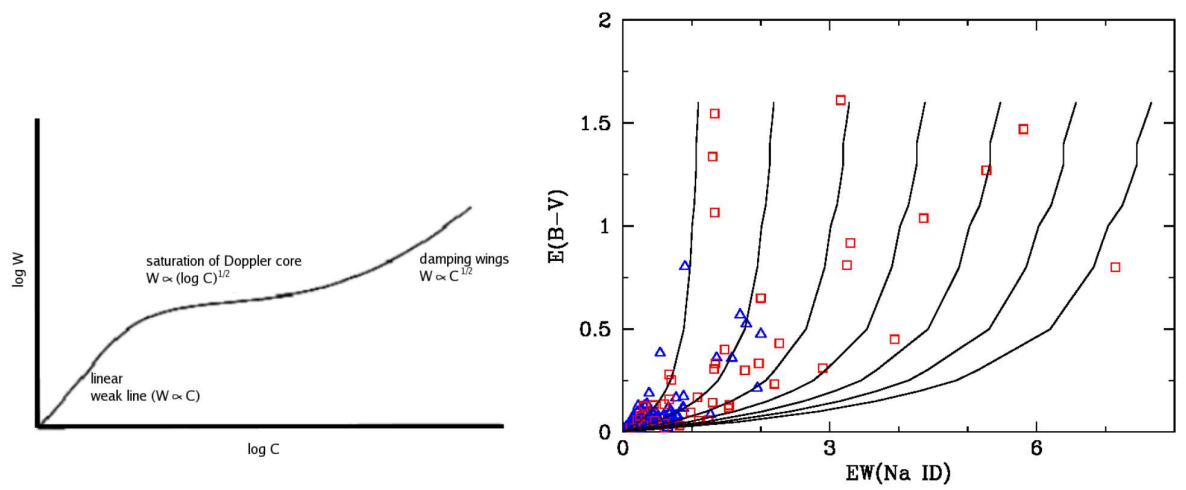

Figure 5: Left: Equivalent line width versus optical depth at the line centre (curve of growth). Note that in our nomenclature $W \equiv \mathrm{EW}$ and $C \equiv \tau_{0}$ [32]. Right: Superposition of curves of growth on the EW(Na ID) vs. $\mathrm{E}(\mathrm{B}-\mathrm{V})$ plane, using several absorbing clouds to cover the area of interest in the $\mathrm{EW}(\mathrm{Na}$ ID) vs. E(B-V) plane.

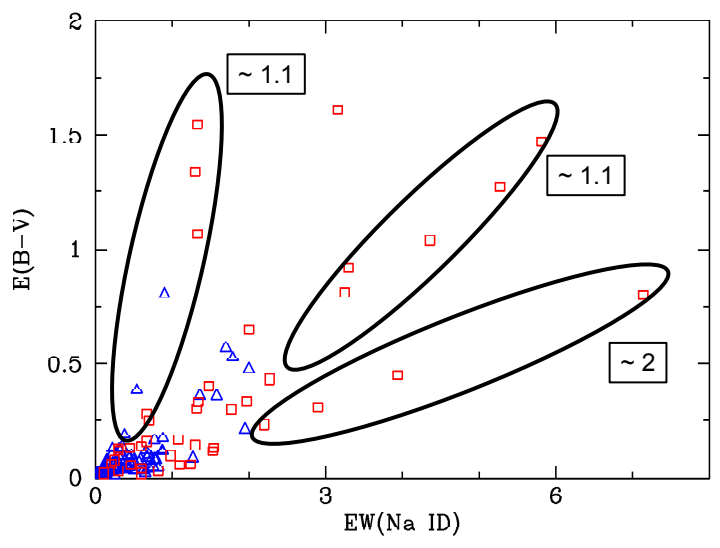

Figure 6: Same as Figure 4 where we have highlighted the region of linear growth (bottom) and those of saturation. The line ratios of the Na ID components are indicated.

is that light from SNe from the lower area passes through diffuse clouds (warmer cloud with low column densities) without saturating the sodium lines which are kept close to linear portion of the curve of growth. The other SNe of larger extinction have sodium lines close to saturation (doublet ratio close to 1.1) which we interpret as due to the lines of sight transiting cooler denser clouds on the more saturated portions of the curve of growth.

This last scenario is therefore able to explain the distribution in the EW(Na ID) $-\mathrm{E}(\mathrm{B}-\mathrm{V})$ plane shown in Figures 4 to 6, but we cannot rule out some influence of the other effects described. Probably the reason for the presence of only three apparently distinct linear relations in the plot 
(Figure 6) was the small sample of highly extinguished SNe. The study of a newly enlarged sample of SNe indicate that the distribution of SNe in the $\mathrm{EW}(\mathrm{Na} I D)$ vs. $\mathrm{E}(\mathrm{B}-\mathrm{V})$ plane is widely scattered. No clear empirical relation between these two quantities can be determined to derived the colour excess (and hence the absorption) from the EW(Na ID) lines. Nevertheless, the plot does provide a lower limit envelope to the reddening as a function of EW, of the form (Figure 7)

$$
E(B-V)=0.11 \times E W(N a I D)-0.01
$$

to indicate that $\mathrm{SN}$ with large EW(Na ID) suffer certainly high extinction.

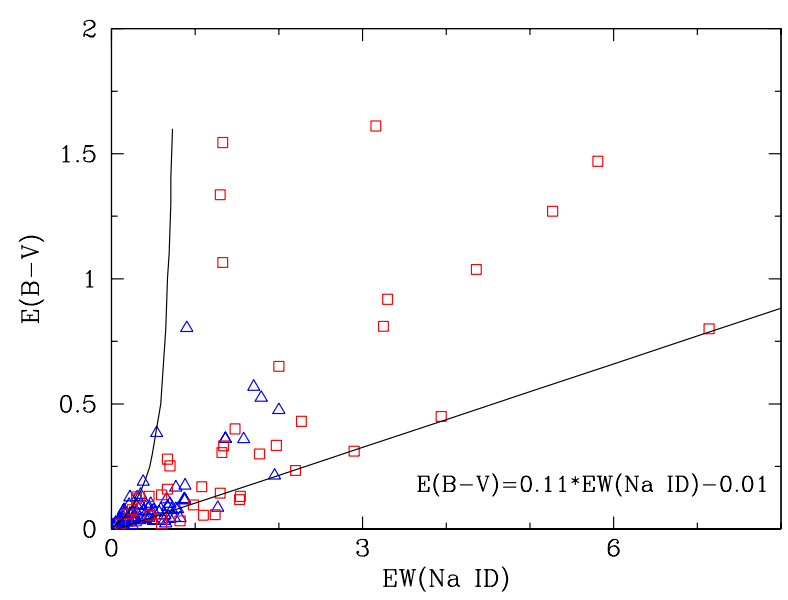

Figure 7: Limits on the plane EW(Na ID) vs. E(B-V). The upper limit is the curve of growth of a single cloud (see text) and the lower limit is reproduced by the relation $\mathrm{E}(\mathrm{B}-\mathrm{V})=0.11 \times \mathrm{EW}(\mathrm{Na} \mathrm{ID})-0.01$.

\section{Conclusions}

As we have seen, from the detailed study of highly reddened SNe Ia such as SN 2002cv, SN 2003cg and SN 2006X we are able to derive the dust properties along the line of sight with a combination of classical photometry and spectroscopy; that is, using the uniformity of the photometric properties of the $\mathrm{SNe}$ Ia we was able to derive reliable values for $\mathrm{A}_{V}$ and $\mathrm{R}_{V}$ of the dust along the relevant lines of sight. In all the three cases of highly extinguished $\mathrm{SNe}$ studied so far, they follow non-canonical extinction laws with lower values of $\mathrm{R}_{V}$ probably due to a small grain sizes which might be the effect of the SN radiation on the local "circumSN" dust or of the peculiar physical conditions in the dust lanes of the host galaxies. Whether this is the case only for high extinction objects is not clear yet. Also we have carried out a new study on the relation of the $\mathrm{EW}(\mathrm{Na} \mathrm{ID})$ vs. $\mathrm{E}(\mathrm{B}-\mathrm{V})$ with a new enlarged sample. Understanding the relation between the physical properties of gas and dust in the direction to $\mathrm{SNe}$ and their effect on the observable $\mathrm{SN}$ is extremely important both for understanding the nature of the SNe explosions and for the calibration of SNe Ia for cosmological use. 


\section{References}

[1] J. C. Weingartner \& B. T. Draine, Dust Grain-Size Distributions and Extinction in the Milky Way, Large Magellanic Cloud, and Small Magellanic Cloud, ApJ 01 (548) 296 [astro-ph/ 0008146 ].

[2] A. G. G. M. Tielens, C. F. McKee, C. G. Seab \& D. J. Hollenbach, The physics of grain-grain collisions and gas-grain sputtering in interstellar shocks, ApJ 94 (431) 321.

[3] K. J. Borkowski \& E. Dwek, The Fragmentation and Vaporization of Dust in Grain-Grain Collisions, ApJ 95 (454) 254.

[4] S. Mattila, W. P. S. Meikle \& R. Greimel, Highly extinguished supernovae in the nuclear regions of starburst galaxies, New Astronomy Reviews 04 (48) 595 [astro-ph/ 0310117 ].

[5] D. Branch \& G. A. Tammann, Type Ia supernovae as standard candles, ARA\&A 92 (30) 359.

[6] D. C. B. Whittet, Dust in the Galactic Environment, Series in Astronomy and Astrophysics, Bristol and Philadelphia 1992.

[7] J. A. Cardelli, G. C. Clayton \& J. S. Mathis, The relationship between infrared, optical, and ultraviolet extinction, ApJ 89 (345) 245.

[8] E. L. Fitzpatrick, An average interstellar extinction curve for the Large Magellanic Cloud, AJ 86 (92) 1068.

[9] K. A. Misselt, G. C. Clayton \& K. D. Gordon, A Reanalysis of the Ultraviolet Extinction from Interstellar Dust in the Large Magellanic Cloud, ApJ 99 (515) 128 [astro-ph/9811036].

[10] L. Bianchi, G. C. Clayton, R. C. Bohlin, J. B. Hutchings \& P. Massey, Ultraviolet Extinction by Interstellar Dust in External Galaxies: M31, ApJ 96 (471) 203.

[11] G. C. Clayton, Extinction in Other Galaxies, in proceeding of Astrophysics of Dust, edited by A. N. Witt, G. C. Clayton \& B. T. Draine, ASP Conf. Ser. Vol. 309, San Francisco 2004, p. 57.

[12] B. T. Draine, Interstellar Dust Grains, ARA\&A 03 (41) 241 [ast ro-ph / 0304489 ].

[13] N. Elias-Rosa et al., SN 2002cv: A Heavily Obscured Type Ia Supernova, MNRAS 08a (384) 107 [0710.4503].

[14] N. Elias-Rosa et al., Anomalous extinction behaviour towards the Type Ia SN 2003cg, MNRAS 06 (369) 1880 [astro-ph/0603316].

[15] N. Elias-Rosa et al., MNRAS $\mathbf{0 8 b}$ in prep.

[16] E. L. Fitzpatrick, Interstellar Extinction in the Milky Way Galaxy, in proceeding of Astrophysics of Dust, edited by A. N. Witt, G. C. Clayton \& B. T. Draine, ASP Conf. Ser. Vol. 309, San Francisco 2004, p. 33 [astro-ph/0401344].

[17] M. Capaccioli, E. Cappellaro, M. della Valle, M. D’Onofrio, L. Rosino \& M. Turatto, Distances of the Virgo and Coma clusters of galaxies through novae and supernovae, ApJ 90 (350) 110.

[18] M. Pozzo, W. P. S. Meikle, J. T. Rayner, R. D. Joseph, A. V. Filippenko, R. J. Foley, W. Li, S. Mattila \& J. Sollerman, Optical and infrared observations of the TypeIIP SN2002hh from days 3 to 397, MNRAS 06 (368) 1169 [astro-ph/ 0602372$].$

[19] P. Goudfrooij, T. de Jong, L. Hansen, H. U. Nrgaard-Nielsen, Interstellar Matter in Elliptical Galaxies - Part Three - Properties of Dust Extinction, MNRAS 94 (271) 833.

[20] M. K. Patil, S. K. Pandey, D. K. Sahu \& A. Kembhavi, Properties of dust in early-type galaxies, A\&A 07 (461) 103 [astro-ph/0611369]. 
[21] A. G. Riess, W. H. Press \& R. P. Kirshner, Is the Dust Obscuring Supernovae in Distant Galaxies the Same as Dust in the Milky Way?, ApJ 96 (473) 588.

[22] L. Wang, Dust around Type Ia Supernovae, ApJ 05 (635) L33 [a stro-ph / 0511003 ].

[23] F. Patat, Reflections on reflexions - I. Light echoes in Type Ia supernovae, MNRAS 05 (357) 1161 [astro-ph/0409666].

[24] F. Patat et al., Detection of Circumstellar Material in a Normal Type Ia Supernova, Science 07 (317) $924[0707.2793]$

[25] J. S. Mathis, W. Rumpl \& K. H. Nordsieck, The size distribution of interstellar grains, ApJ 77 (217) 425.

[26] R. Barbon et al., Type IA supernova 1989B in NGC 3627, A\&A 90 (237) 79.

[27] U. Munari \& T. Zwitter, Equivalent width of NA I and K I lines and reddening, A\&A 97 (318) 269.

[28] M. Turatto, S. Benetti \& E. Cappellaro, Variety in Supernovae, in proceeding of From Twilight to Highlight the Physics of Supernovae, edited by B. Leibundgut, W. Hillebrandt, Springer-Verlag, Berlin 2003, p. 200 [astro-ph/0211219].

[29] N. Elias-Rosa et al., $A \& A \mathbf{0 8 c}$ in prep.

[30] R. Barbon, V. Buondí, E. Cappellaro \& M. Turatto, The Asiago Supernova Catalogue - 10 years after, A\&AS 99 (139) 531 [astro-ph/9908046].

[31] P. Lira, M. S. Thesis, University of Chile, 1995.

[32] R. L. Bowers \& T. Deeming, Astrophysics Vol. 1,2, Jones and Bartlett Publ., Boston, 1984.

[33] L. Jr. Spitzer, The Distribution of Interstellar Sodium, ApJ 48 (108) 276. 